\title{
Manga Generator, a future of interactive manga media
}

\author{
Invited Talk Paper
}

\author{
Akihiko SHIRAI \\ Kanagawa Institute of Technology \\ shirai@mail.com
}

\begin{abstract}
This article contributes to the realization of an immersive role playing manga generating advertising system named "Manga Generator". The project began as a student virtual reality project and had underwent various collaborations with professional manga artists and governmental agencies. It was exhibited at many international exhibitions and is currently a permanent exhibition in a museum. The latest version "MGV" is a new advertising entertainment system that enables up to two players to engage in role playing interactive manga while allowing other audiences to see advertisement video simultaneously using multiplex hidden image technology "ExPixel". In this research, we combined interactive comic generating system with full body motion detection and real time image processing technologies. It can attract non-audiences who are not interested in the video announcements thanks to players playing Manga Generator.
\end{abstract}

\section{CCS Concepts}

$\bullet$ Human-centered computing $\rightarrow$ Empirical studies in interaction design;

\section{Keywords}

role-playing, interactive, Kinect, motion sensor, manga, multiplex hidden image, comic, advertising, digital signage

\section{INTRODUCTION}

In the past several decades manga has spread from Japan to the rest of the world enriching the lives of countless people with their rich stories and unique art styles. Despite of the many existing manga related products out there such as anime and licensed video games, there exist few applications that allow players to physically interact with their own manga fantasies.

\section{MOTIVATION FROM STUDENT COM- PETITION PROJECT}

Permission to make digital or hard copies of all or part of this work for personal or classroom use is granted without fee provided that copies are not made or distributed for profit or commercial advantage and that copies bear this notice and the full citation on the first page. Copyrights for components of this work owned by others than the author(s) must be honored. Abstracting with credit is permitted. To copy otherwise, or republish, to post on servers or to redistribute to lists, requires prior specific permission and/or a fee. Request permissions from permissions@acm.org.

MANPU '16, December 04 2016, Cancun, Mexico

(C) 2016 Copyright held by the owner/author(s). Publication rights licensed to ACM. ISBN 978-1-4503-4784-6/16/12 . \$ \$15.00

DOI: http://dx.doi.org/10.1145/3011549.3015156
This project "Manga Generator", began its development by an international student during the virtual reality contest "IVRC", International collegiate Virtual Reality Contest in 2012.

The original motivation was proposed by Mr Yukua KOIDE in Shirai Lab. He was a master student with technical and fine arts skills who read manga extensively. His love for manga led to him envisioning himself as characters in different manga scenarios. This idea was finally realized in IVRC, a virtual reality contest with 23 years of history. The first IVRC was established and held in Tokyo as a part of the International Conference on Artificial Reality and Telexistence conference (ICAT). In ACM SIGGRAPH, 21 projects were accepted by the Emerging Technologies (a.k.a E-Tech) committee[5].
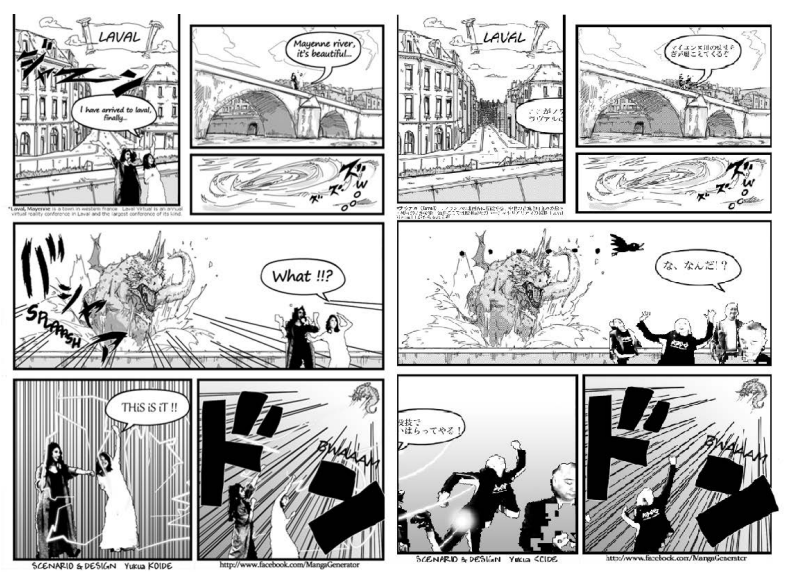

Figure 1: Examples of generated manga pictures by "Manga Generator" system in Laval Virtual. Generated images are not same in each generated pictures thanks to AccuMotion and KineMotion algorithms.

\section{TECHNOLOGY}

\subsection{Manga Generator}

Manga Generator is an interactive role playing manga generating system that uses Microsoft Kinect to capture the body motion of the players and juxtaposes the these images together with speech bubbles and background effects onto manga panels to create highly personalized manga featuring captured images of the players. By using Kinect to de- 
tect the skeletal structure of the players, Manga Generator was able to place speech bubbles and background special effects in appropriate locations in relation to the player's body. Other hardware involved in the making of Manga Generator include a projector that projects the game onto a screen, a speaker that broadcasts audio cues of the game to the players, a printer that prints out manga stripes featuring the players' images, and a pc where all the other hardware except for the screen connects to.

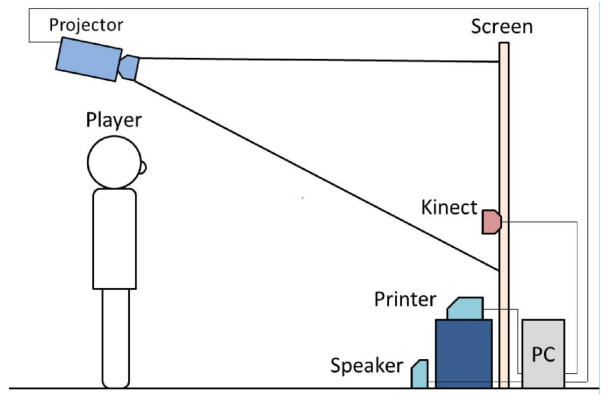

Figure 2: Main components of "Manga Generator", in the first version

\subsection{Sensory motor play generates original im- ages}

Manga Generator has been tested in public and exhibitions and it was shown that by having a player subjectively acting out the different scenarios, we could place an immersive role playing posing game into the manga world and automatically create manga reflecting the personality of the player. What sets this system apart from simple shader and comic chat which output manga-like visuals is that our system realized a game system that gives the player freedom to use their imagination without interfering with the world view of the original work. Essentially we have created a platform that offers the sensation of freedom in a controlled storytelling environment.

The Manga Generator system is also equipped with body gesture sensors and can generate different backgrounds and special effects according to the emotions conveyed by the players' overall gesture. Depends on how vivid and action oriented the players' gestures are, each player is given a posture score that in turn decides how action packed the background effects are.

KineMotion[2] algorithm is a subset of AccuMotion[4] algorithm which is motion analysis algorithm for real time interaction using Kinect. The posture score is calculated by combining a series of inner products drawn from the bending degree of the joints, Skeleton information, and kinematics obtained from Kinect. The inner products take its range as -1 to 1 and a following formula can obtain enhanced sum of inner product of each focused joints: $F=$ $0.15 f_{\text {elbL }}+0.15 f_{\text {elbR }}+0.25 f_{\text {axi } L}+0.25 f_{\text {axi } R}+0.2 f_{\text {pel }},(1.0 \leq$ $F \leq 1.0)$. Once all some interested inner products have being obtained, $F$ can be used to the similarity from the target postures. This evaluated score can apply for different background effects depending on which emotional threshold the score reaches. The higher the score the more intense the manga background effects are. This makes our Manga Generator highly replayable with each play-through generating a different result.

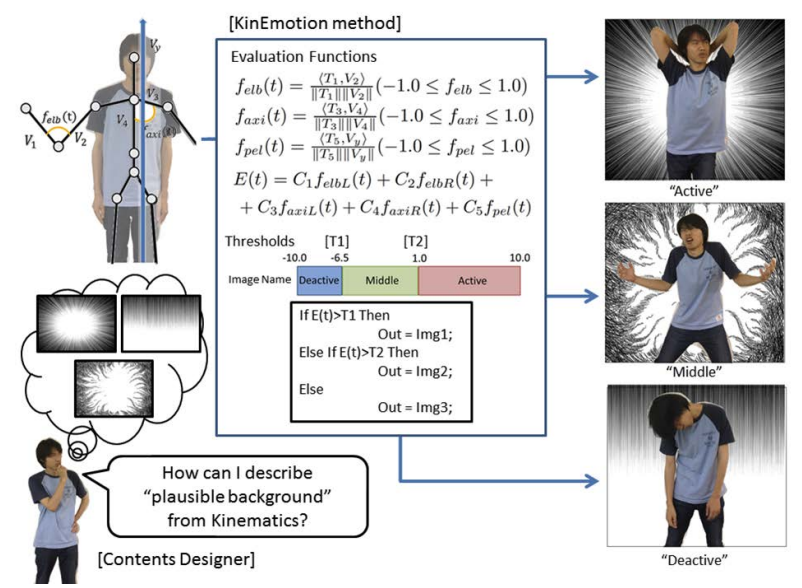

Figure 3: Main components of "Manga Generator", in the first version

Our Pixel Shaders can generate cartooned images in real time. The players can be free but they have a chance to express their emotion in manga pieces, that is a state of arts of this project. Players can feel their own original senses by their entire body expression and it generates original images by their creativity and curiosity. We think it is a effects of sensory motor play, one of an elemental human play for all kind of people.

\section{RESULTS}

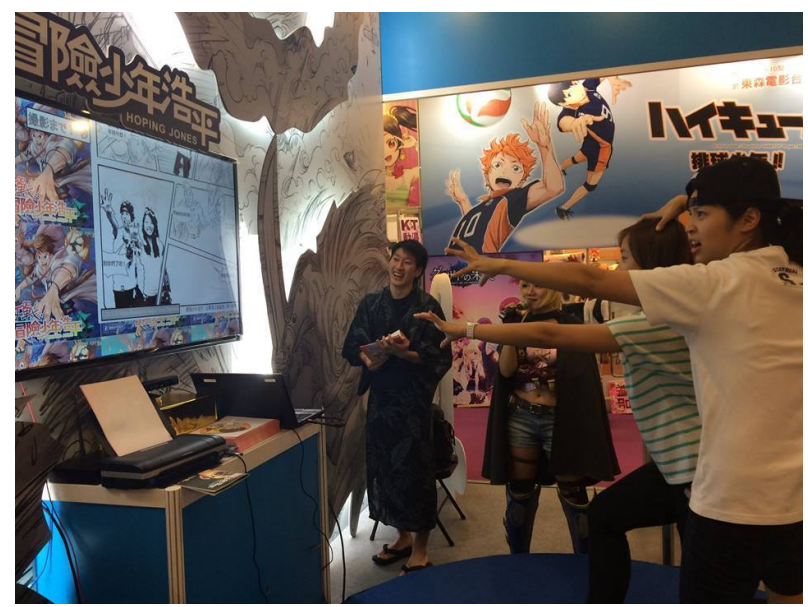

Figure 4: "Hoping Jones" version in Taiwan Manga Expo 2015.

\subsection{Internationalization}

Manga Generator won the 3rd place in IVRC2012, an immersive posing role playing game that reflects the personality of the player who acts out the part of the hero, using pre-installed speech bubbles, backgrounds, effects, and emotion recognition that, when combined, forms the manga. The project has many international versions and awarded in Laval Virtual in France. More than 200 projects have been realized through the past IVRC in Japan. Typically 
3 people work in each projects, therefore, we can estimate that over thousands persons have joined in this competition (In global, here are more participants from France and the U.S.A). Often an IVRC students becomes a staff in next edition, experienced staff becomes an executive committee member and/or an educators in academic virtual reality domain.

\subsection{Manga Generator as a tourism resources}

The updated version has collaborated with the Tottori Prefecture, which is promoting tourism resources with some famous manga authors, Shigeru MIZUKI, Gosho AOYAMA and Jiro TANIGUCHI[7].

\subsection{Collaboration with manga authors}

The project team had collaborated with famous Japanese manga authors such as "Gegege no Kitaro" by S. MIZUKI, "Detective Conan" by G. AOYAMA and "Haruka-na Machihe" by Jiro TANIGUCHI, youtu.be/HOcP1V13Kb0) and "Hoping Jones" from Taiwan. Shigeru MIZUKI had past away in 2015 but the system can generate his manga if the Tottori Manga Kingdom (Tottori Prefectural Government) authorized it.

\section{ADVANCEMENTS}

\subsection{Player analysis in permanent exhibition}

It is also installed in a museum named TEPIA in Tokyo as a permanent exhibition. As a scientific research, we obtained the authorization to take photos and images from public visitors. In 2014, it had been played by 41,525 visitors in 267 days. The obtained players' posture with a finisher situation are useful to analyze which contents are suitable for the player persona (age, group). It might be a good seed database for "combination persona" that means grouped visitors.

\subsection{Contents toolkit using Photoshop script}

To make various contents, we have a toolkit using Adobe Photoshop script which supports the exportation of PNG files with parameter files for interactivity. The natural user interface (NUI) is made to choose several contents for automatic operation. In TEPIA, when the system is alone, the system shows promotion movie then if any visitor comes, the

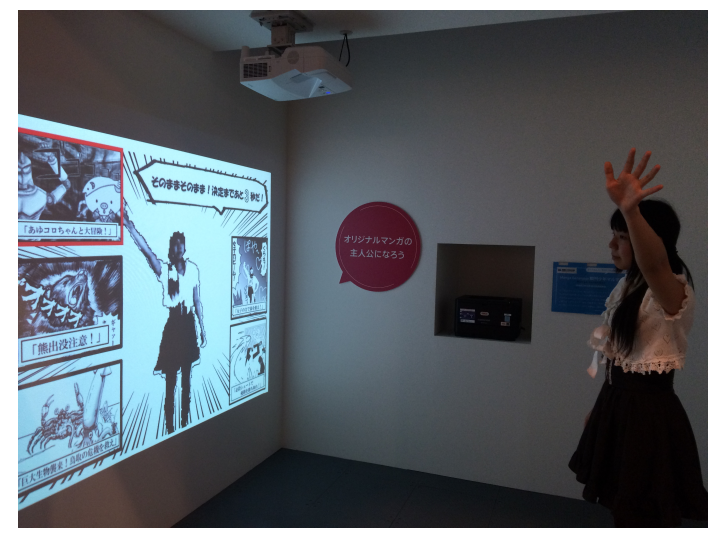

Figure 5: TEPIA permanent museum exhibition.

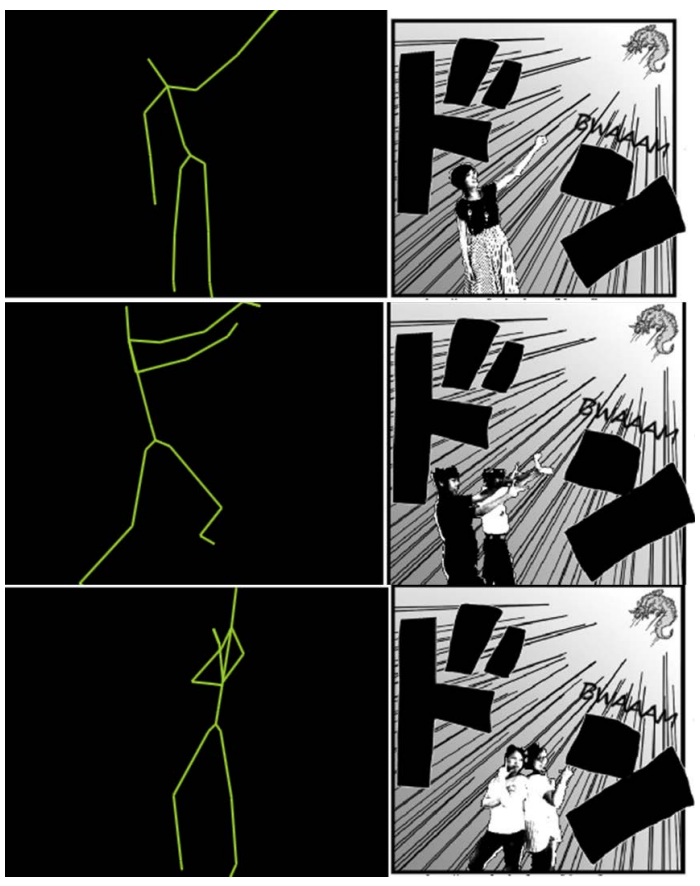

Figure 6: Examples of obtained players' posture with a finisher
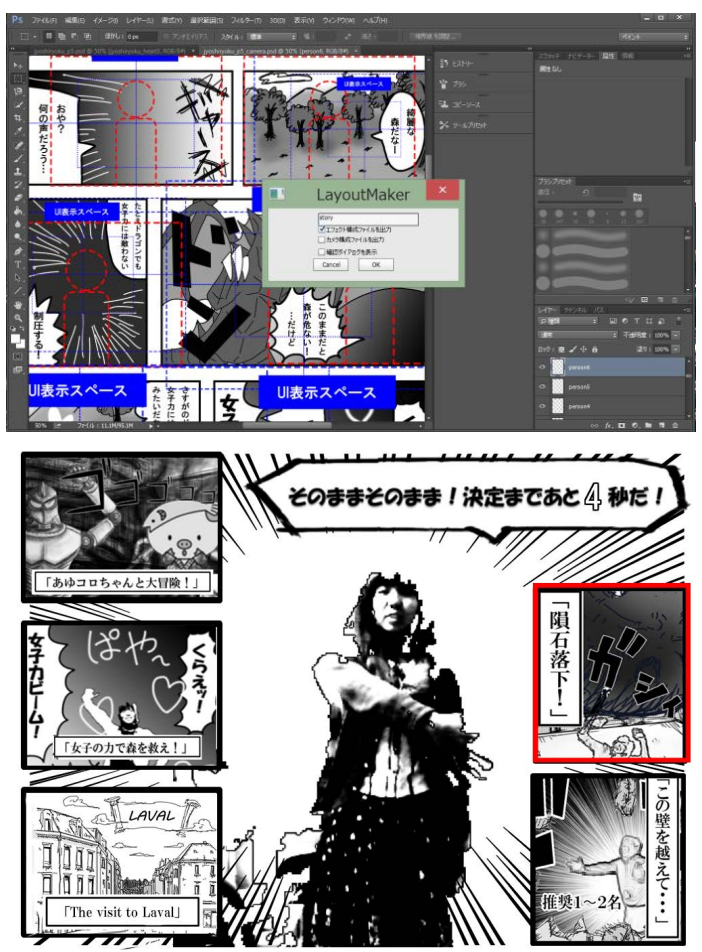

Figure 7: A toolkit for Manga Generator contents / Menu and its user interface 
experience starts automatically. The system counts which contents had been chosen and concludes how it had been played by whom.

\section{MGV: MULTIPLEX DIGITAL SIGNAGE VERSION}

The Manga Generator Visualization system, which combines Manga Generator and ExPixel FPGA is a hybrid of both interactive entertainment and video systems that satisfies both players and observers alike with two distinctive contents. MGV allows players to be immersed into manga worlds on the hidden channel using special polarized glasses while audiences not wearing glasses get to watch a separate video channel on the naked eye channel. This we believe will satisfy people who are interested in interactive role playing game and video footage at the same time and encourage interaction between audiences by having everyone present at the same room. Since all hardware required to run the system are already commercially available, the MGV can be cheap to purchase and easy to install at public places and homes. Furthermore MGV is equipped with Unity plug-ins allowing developers to develop customize contents with ease. We are hoping that content developers and players alike will be able to enjoy their manga contents with MGV.

"ExPixel" is a multiplex hidden image technology [1] utilizing polarization filters and commercially available Liquid Crystal Display to allow viewers to see different images using special polarized glasses. "ExPixel FPGA"[6] improves upon "ExPixel" by being able to generate multiplex hidden images by attaching 2 HDMI input and 1 HDMI output. "ExPixel FPGA" also supports HD digital video sources (mainly designed as $(1920 \times 1080 \times 60 p)$. ExPixel core algorithm had been implemented by look up table (LUT) to obtain inversed gamma on ATLYS Spartan-6 XC65LX45 with 2 inputs and 1 output. Plugged sources are decoded and combined to form a striped image for line-by-line 3D. The image is performed by ExPixel core and then an encoder puts a HDMI output signal with correct period signal. The core just needs 1 frame buffer to perform entire process. It had been implemented by FPGA internal BlockRAM as a line buffer and Xlinx's Memory Interface Generator (MIG) with DDR2 SDRAM as a frame buffer.

\section{CONCLUSION}

MGV is one such technology that enables players to become the protagonists of their own manga adventures. In this project we integrated two entertainment technologies: Manga Generator[3] and "ExPixel" FPGA to create MGV the digital signage integrated Manga Generator using Multiplex Hidden Imagery. On top of acting as a role playing manga system, MGV also doubles as an advertisement system by using multiplex hidden imaging technology to advertise merchandises, creating a system capable of providing entertainment while advertising for commercial products simultaneously.

We have proven from experiments that ExPixel can be used to combine a variety of media and platforms together. We have succeeded in combining Xbox 360 and Wii U game consoles together as well as integrating game councils with video footage, still images, and other media. Furthermore, we have incorporated directional audio system into ExPixel, allowing audiences to experience both the visual and audio

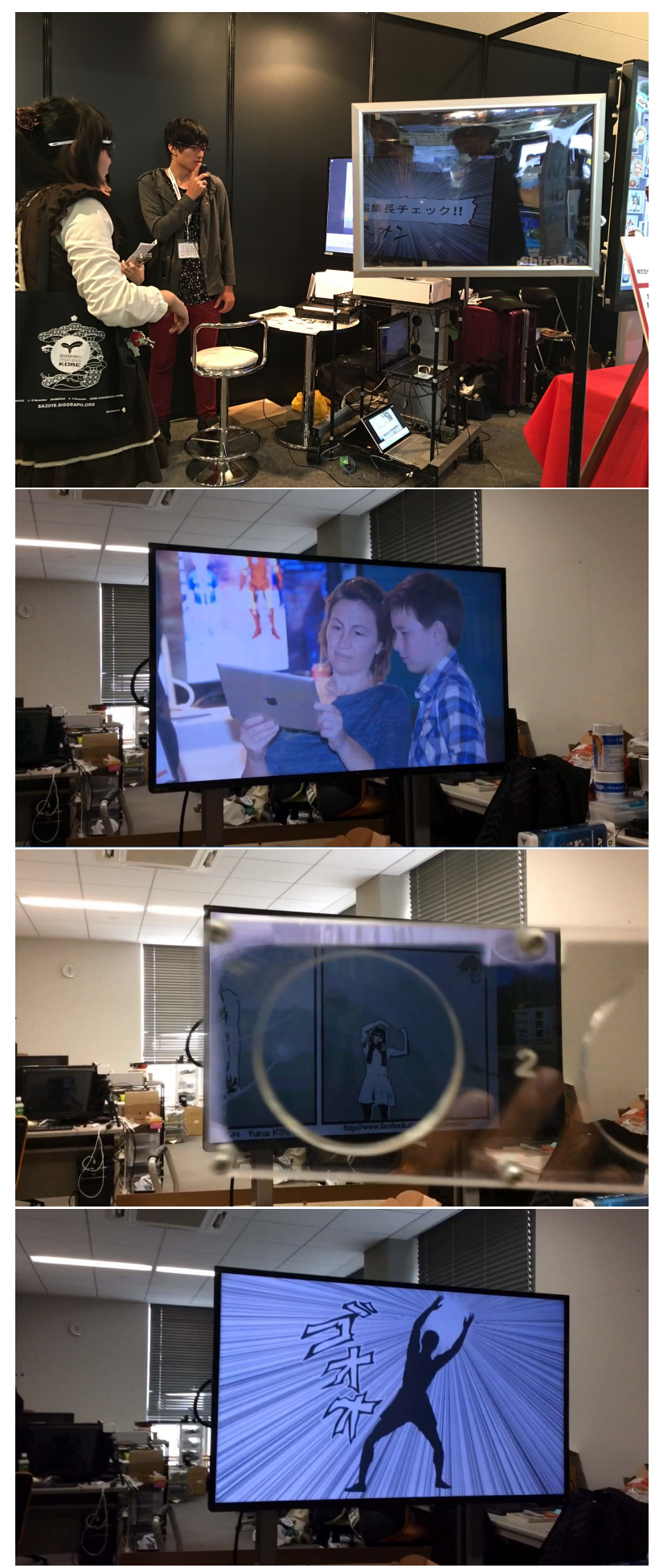

Figure 8: Examples of MGV using ExPixel FPGA and passive stereo $3 \mathrm{D}$ display. Two images could be shown by polarization filter simultaneously. When viewed with naked eyes one gets to see slide-show like advertisements while visitors wearing glasses get to watch and play the interactive role playing manga game. When manga generator players play the game and strikes a pose, advertisement viewers will see different contents popping up. 


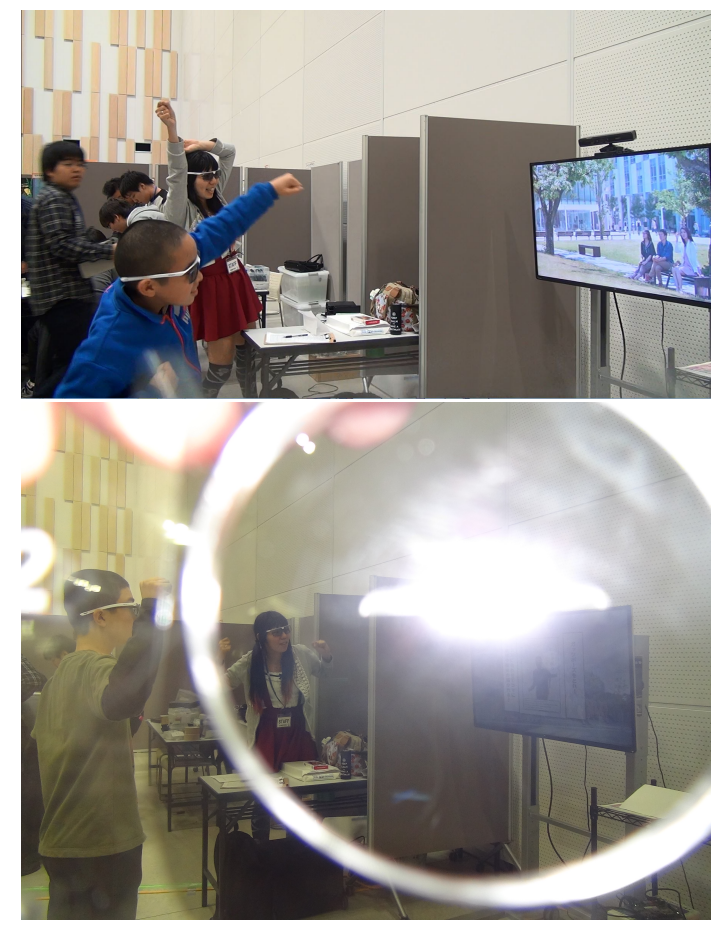

Figure 9: Installation using MGV system, upper photo is players behavior in public demo, the screen shows a promotion video as a digital signage. Lower photo shows a gaming visual which is shown by polarization filter using "ExPixel" technology.

aspects of a content without interference from unrelated contents. The special polarized glasses do not require electricity to run and are easy and cheap to manufacture. The result is a system that allows two contents to be enjoyed simultaneously on the same Liquid Crystal Display screen without spending a fortune as all hardware are already commercially available.

\section{FUTURE RESEARCH OF INTERACTIVE MANGA MEDIA}

As the development of Manga Generator reaches its maturity, a future of interactive manga media exists using new media technologies like virtual reality, natural human interfaces, real time image processing, and printing. Now the project will undergo behavior analysis, understanding, recognition, retrieval, and analysis. The output media will not be limited only by a paper media but also by social media like Twitter and/or Facebook. Scenario making and its narrative will be dynamic and the toolkit will be faciliated for user generated contents. Deep learning and its learning filters will be useful to enhance the result and it will be also useful to propose actual manga purchaces from player's postures. We expect the project will become a fronteer of this research domain, "Manga Generator", for further researches.

\section{ACKNOWLEDGMENTS}

Author would mention thanks to all contributors of Manga Generator project, Yukua KOIDE, Wataru FUJIMURA, Yuto NARA, Genki KUNITOMI, Kosaku OGAWA, Ayumi ANDO,
Ryo OKAMOTO, Hisataka SUZUKI and Rex HSIEH.

\section{REFERENCES}

[1] Y. Littfass, Y. Koide, H. Suzuki, and A. Shirai. "scritter" to "1p2x3d": Application development using multiplex hiding imaging technology. In Proceedings of the 2014 Virtual Reality International Conference, VRIC '14, pages 21:1-21:4, New York, NY, USA, 2014. ACM.

[2] Y. Nara, W. Fujimura, Y. Koide, G. Kunitomi, and A. Shirai. Kinemotion: Context controllable emotional motion analysis method for interactive cartoon generator. In ACM SIGGRAPH 2013 Posters, SIGGRAPH '13, pages 75:1-75:1, New York, NY, USA, 2013. ACM.

[3] Y. Nara, G. Kunitomi, Y. Koide, W. Fujimura, and A. Shirai. Manga generator: Immersive posing role playing game in manga world. In Proceedings of the Virtual Reality International Conference: Laval Virtual, VRIC '13, pages 27:1-27:5, New York, NY, USA, 2013. ACM.

[4] T. Sakai, W. Fujimura, S. Robert, T. Kosaka, and A. Shirai. Accumotion: Intuitive recognition algorithm for new interactions and experiences for the post-pc era. In Proceedings of the 2012 Virtual Reality International Conference, VRIC '12, pages 15:1-15:5, New York, NY, USA, 2012. ACM.

[5] A. Shirai, J. Nishida, D. Sato, K. Sato, H. Takatori, and S. Tachi. Innovation in the age of virtual reality through organizing international student competition. In SIGGRAPH Asia 2015 Symposium on Education, SA '15, pages 8:1-8:3, New York, NY, USA, 2015. ACM.

[6] H. Suzuki, R. Hsieh, R. Tsuda, and A. Shirai. Expixel fpga: Multiplex hidden imagery for hdmi video sources. In ACM SIGGRAPH 2015 Posters, SIGGRAPH '15, pages 71:1-71:1, New York, NY, USA, 2015. ACM.

[7] P. G. Tottori. Call for entries to 5th annual manga kingdom tottori international comic art contest. Press Release, Kyodo JBN, 2016. 